\title{
Analysis on the Utility Function of Consumers' Choice of Internet Financial Products En Deng ${ }^{12}$, Ming Zhao ${ }^{2 *}$
}

\author{
${ }^{1}$ School of Economics and Trade, Hunan University, Changsha, Hunan, 410082; \\ ${ }^{2}$ Hunan Radio and Television University, Changsha, Hunan 410004 \\ *The Correspondence Author
}

\begin{abstract}
The Internet economy era is an ear with "surplus information but scarce attention". When transmitters of brands in the Internet finance industry seek a technical method to match customers' scarce attention with massive information, they can know what product elements are of customers' concern and what factors affect customers' choice, so they can easily understand and accurately grasp the information that can move customers. Thus it can be seen that todays' customers' choice of Internet finance brands is subject to the influence of various factors. After obtaining the information of customers' interest, customers can understand and compare the brands to get the maximum utility portfolio and pursue the maximum individual utility of customers with certain range of brands according to the product attributes and brand transmission attributes and then make the consumption decisions. Starting from the real choices of customers in China's Internet finance industry, this paper selects to study the factors affecting customers' consumption choices in the current Internet finance industry from the perspective of brand transmission and reconstruct to consider the utility function of brand transmission factors based on this so as to provide references for researches and real business managers of brand transmission.
\end{abstract}

Keywords: Internet finance industry; Customers' choice; Utility function

\section{浅析消费者选择互联网金融业产品的效用函数}

$$
\text { 邓恩 }{ }^{1,2} \text { ，赵明 }{ }^{2 *} \text {, }
$$

（1．湖南大学经济与贸易学院，湖南长沙 410082;

2. 湖南广播电视大学, 湖南长沙 410004

*通信作者)

摘要: 互联网经济时代是一个 “信息过剩而注意力稀缺” 的时代。当互联网金融业品牌传播者寻找一种技术方式将潜在 客户稀缺的注意力同海量的信息匹配到一起时，能知道消费者在关注哪些产品要素、消费选择受哪些因素影响，那么就很容 易了解并精确把握哪些信息可以打动消费者。可见，当今消费者对互联网金融业品牌的选择受多种影响因素的影响，在获取 到消费者感兴趣的信息后, 消费者将对品牌进行了解和比较得出最大的效用组合, 在一定品牌范围内按产品属性、品牌传播 属性等因素来追求消费者个人的效用最大化，并做出消费决策。该文从中国互联网金融业消费者选择的现实出发，选取品牌 传播视角来研究当前互联网金融业消费者消费选择的影响因素, 并以此为基础来重构考虑品牌传播因素的效用函数, 为品牌 传播的研究者和实际业务管理者提供参考。

关键词: 互联网金融业; 消费者选择; 效用函数

中图分类号: F49.1 文献标志码: A

\section{引言}

传统主流经济学对消费者选择行为的分析主要考虑商品价格既定情况下，不同商品数量带给消费者的 效用，当消费者投入的单位货币实现的不同商品边际效用实现均等时，且等于单位货币的边际效用，则实 
现了消费者均衡, 即效用最大化原则。这种理论分析借助无差异曲线和预算线非常巧妙地解决了基数效用 论中不同消费者之间效用不可比较的难题, 构成了经济学关于消费者选择行为理论的主体。但此理论没有 考虑到互联网金融业产品的品牌传播因素，消费者对不同品牌的信息获取并不是均等机会和无成本的，特 别是在当今中国商品经济日益发达、互联网金融业发展迅猛、互联网金融品牌种类繁多、品牌信息相对过 剩、消费者注意力资源有限的背景下，互联网金融业消费者对品牌的选择行为更具复杂性，不是简单考虑 商品的效用、数量、价格来进行消费选择的。因此, 我们很有必要对影响消费者品牌选择的影响因素进行 分析，并以此为基础重构互联网金融业消费者选择产品的效用函数。

\section{1 互联网金融业消费者品牌选择的影响因素}

\section{1 品牌传播}

互联网金融业作为结合传统金融功能与互联网技术的一个新兴行业，其品牌价值传播的有效性将直接 影响着消费者对该品牌金融产品的选择性。一般而言，决定该品牌传播效果的因素有：品牌推广的传播媒 介、品牌地位、品牌声誉等。其中, 不同的传播媒介, 其传播的时效性、有效性和渠道也不同。传统的传 播媒介为报纸、书刊、广播、电视等。如今, 随着微博、微信等网络媒体、新兴媒体的发展, 在更新换代、 信息快速更迭的现代社会，高时效、高质量、多渠道的新媒体已成为大多数品牌推广的重要媒介。品牌在 本行业的地位也是一个重要的传播影响因素。不同品牌其注册资本、公司资历、社会关系和商业模式、专 业能力的不同，将产生不同的品牌地位，影响着消费者对其金融产品的选择。而在某些社会事件中，品牌 行为所产生的品牌形象和品牌声誉甚至品牌事件等同样将会影响着消费者的决策。

\section{2 产品属性}

消费者在进行消费选择时, 产品属性是影响其决定的最重要因素之一。产品属性一般包括产品名称、 产品的抗风险性、往期收益、产品价格和获取该产品的便捷程度等。产品名称是消费者接触该产品的第一 印象，随不同消费者的喜好和偏好不尽相同，但一个高雅且接地气的产品名称将更容易获取消费者的第一 好感。产品的抗风险性即产品承担风险的能力, 决定了该产品的收益波动范围, 抗风险能力越高、产品收 益波动范围越小的产品在交易过程中更易受消费者青睐。产品往期的收益价格对消费者而言, 是对该产品 的一个更直观的评估, 往期收益越高、收益越稳定或呈现出更优收益走势的产品, 消费者有足够的理由去 选择它。产品价格将成为消费者的一个相互选择的门槛, 对于互联网金融产品这种新兴产品而言, 不同时 期、不同产品有着不同目的和不同的定价策略。在进行推广的初期, 一些低端产品为了留住消费者、构建 基础的消费者群体与其建立联系, 一般采用产品定价较低的策略, 从而给消费者一个合适的、较低的 “尝 鲜” 成本。而在发展的中后期, 为了对消费者进行分层分阶级进而提供针对性服务和发展, 一些高端产品 将会设置不同梯度的定价, 以使企业利润达到最大化。此外, 一般消费者由于缺少专业人士的指导, 自己 获取产品信息的能力、渠道和时间有限，考虑到精力和时间成本，消费者更乐意选择购买和使用更便捷、 更灵活的产品。

\section{3 个人因素}

消费者的个人因素也是影响消费者决策的重要因素。而个人因素包括消费者个人期望和需求、个体差 异等。不同消费者在不同场景下的个人期望和需求不同，为了了解和满足消费者的不同期望和需求，品牌 如何与消费者之间建立有效的联系和沟通就显得格外重要。消费者个体差异包括消费者的性别、年龄、投 
资理念、接受新兴和高风险产品的能力、及受教育程度和消费能力等。男性和年轻的消费者群体, 其思维 方式、消费习惯和对高风险的接受能力, 决定了其更倾向于选择回报收益较大的高风险产品。同时, 受教 育程度越高、消费能力越强的消费者所能选择的互联网金融产品范围越大，因为其个人能力突出的优势使 其拥有更多的渠道和方法去获取更多的产品和更专业的多方位信息，从而使该消费者群体拥有更多的选择 性、更易做出达到自己期望、满足自己需求的决策。

\section{4 微观环境}

互联网金融行业与传统金融行业相比，利用了互联网技术，使其金融产品能够在网络平台呈现，让消 费者拥有一个更加透明、有效率的选择空间。而在这样的方式下, 如何保持良好的竞争力, 就需要对其微 观环境下功夫。主要为互联网金融产品网络平台的设计影响等。网络平台对互联网金融产品而言, 是工具 也是机遇，尤其是其 APP、网页的制作、端口的设计等，是否简洁明了、美观大方、突出重点和特点，都 将会影响着消费者对互联网金融网络平台和金融产品的印象，从而进一步影响其决策。

\section{5 宏观环境}

对于互联网金融业这种新兴行业而言，宏观环境也是对其发展的一大考验。宏观环境方面包括国家政 策、银行政策、消费者接触到互联网金融产品的难易程度、互联网金融产品在市场上的容纳度等。2015 年 7 月 18 日，人民银行等十部门联合发布《关于促进互联网金融健康发展的指导意见》(银发〔2015〕221 号)。《指导意见》按照 “鼓励创新、防范风险、趋利避害、健康发展” 的总体要求, 提出了一系列鼓励创 新、支持互联网金融稳步发展的政策措施, 积极鼓励互联网金融平台、产品和服务创新和发展。这一政策 的发布无疑是给市场发布了一个信号, 鼓励并倡导互联网金融的创新、稳步发展。对于消费者购买互联网 金融产品决策而言，起到了一个积极正面的作用，这使得互联网金融产品在市场上具有更大的容纳度，而 消费者也能更加容易接纳并消费自己有兴趣的互联网金融产品。

\section{6 群体参照}

消费者在实施购买决策时，大多有一种从众心理。其品牌是否为大众已接受的品牌、是传统金融企业 的互联网金融大品牌还是新兴的小品牌、该品牌在消费者群体中的口碑如何、是否有已购人员的推荐等, 这都将很大程度地影响着消费者的购买决策。一般而言，传统金融行业结合互联网技术的这种大品牌和银 行互联网金融衍生物较之一些融资较少、起步较晚的小互联网金融品牌更具有群众基础、更受大众欢迎。 但某些后起之秀的品牌当其足够优秀且拥有了一定的口碑、经过已购者的口口相传、互相推荐, 也是非常 具有生机和发展潜力的。此外, 消费者和已购人员之间的亲疏关系也直接影响着其受信任度, 与已购者的 关系越亲近，则对其言论和推荐品牌的信任度则越高，从而实施购买决策的可能性越大。

\section{7 品牌服务}

对于互联网金融业品牌的经营，除去产品本身的价值，其服务的重要性也是不言而喻的。品牌售前售 后的服务质量优劣也将会对消费者最后的决策做出重要的影响。其中, 售前服务主要指消费者的购买体验, 如果消费者在购买中产生了比较愉悦的购物体验, 这不仅会影响消费者本次的最终消费决定, 也将会成为 消费者下次是否依然购买的考虑因素。而售后服务主要在于商家对于消费者的一种承诺, 是一种售后保障, 这意味着该互联网金融品牌所愿意替消费者分担的风险和对自身产品的一个自信度。一般优质的品牌所提 供的售前售后服务都会是更优质、更替消费者着想，也更能打动消费者的。 


\section{8 广告劝说}

在一定程度上，品牌广告劝说力将会影响消费者决策。其包括广告投放渠道、广告制作效果、广告策 划创意等方面。广告投放渠道的不同, 其平台的可信度和传播力也不同, 对消费者的劝说力度都会有着不 同的影响。可信度越高、传播力越大的平台, 其能起到的劝说作用越明显, 消费者从而产生消费的可能性 越高。广告视频制作、文案创意等效果的呈现, 对消费者也是有着巨大的吸引力和说服力。越优质的广告 方案, 在消费者心中越能留下良好的品牌印象, 从而为消费购买该品牌产品产生了一个积极的影响。而广 告劝说所采取的方式十分重要, 其是否能够达到品牌自定的预期效果、是否能恰好满足消费者的偏好非常 关键, 比如明星代言人的选择、节目的冠名赞助等情形。优秀的明星代言人, 其本身的形象气质是有助于 品牌的传播和发展、能够与品牌的定位相契合, 能够向消费者表达出该品牌产品优于其他品牌产品的独到 之处。这有助于大多数感性消费者对该品牌拥有一种亲近感, 使其对该品牌产生特殊偏爱, 从另一方面 建立情感联系，从而产生消费。

\section{2 互联网金融业消费者品牌选择的效用函数}

\section{1 消费者品牌选择的 Logit 效用函数及其原理}

Logit 效用函数最早于 20 世纪开始逐步运用到经济学等学科, 其全称为 Logistic 回归模型, 是研究 人的选择行为过程的计量经济模型, 属于离散选择模型的一种。2000 年诺贝尔经济学奖就颁发给美国芝加 哥大学的詹姆斯・赫克曼和加州大学伯克利分校的丹尼尔・麦克法登, 以表彰他们在离散选择模型等微观计 量经济学领域所作出的贡献。1977 年开始, 越来越多的研究者运用 Logit 模型来分析定性数据, 特别是模 拟消费者的消费选择行为和结果。

Logit 模型是从人们行为的因果关系入手, 考虑如果能考察到所有影响一个选择行为的各类因素及其 作用机制, 则能够在特定条件下用模型进行预测下一次选择行为的发生。由于要洞察所有因素是不可能实 现的, 因此我们将能观测到的影响因素 ( $\left.x_{i}\right)$ 及不能观测到的因素 (处理为随机变量 $\varepsilon$ ) 结合起来考虑, 就得到一个抽象行为函数 $\mathrm{y}=\mathrm{f}\left(\mathrm{x}_{\mathrm{i}}, \varepsilon\right)$, 其中, 在能够观测到的因素 $\mathrm{x}_{\mathrm{i}}$ 下, 选择行为出现的概率就是该行 为函数的随机变量 $\varepsilon$ 的概率。用条件概率公式表示如下:

$$
\mathrm{P}(\mathrm{y} \mid \mathrm{x})=\mathrm{P}(\varepsilon \text { s.t. } \mathrm{f}(\mathrm{xi}, \varepsilon)=\mathrm{y})=\int \mathrm{I}[\mathrm{f}(\mathrm{xi}, \varepsilon)=\mathrm{y}] \mathrm{f}(\varepsilon) \mathrm{d} \varepsilon
$$

\section{2 消费者品牌选择的关键影响因素-一产品性能和品牌传播}

Logit 效用函数运用到互联网金融业消费者品牌选择行为分析上, 能刻画出互联网金融产品的产品属 性、品牌传播属性等各类因素对消费者品牌选择行为的影响。互联网金融业消费者是否选择某个品牌存在 概率大小, 并以此来安排自己的购买决策。可采用效用满足程度来衡量某一互联网金融品牌对消费者的吸 引力。互联网金融品牌对消费者效用满足的程度受个人因素、宏微观环境、产品属性、广告劝说、感知风 险、成本与收益、产品或服务的功能、品牌传播等多因素的影响。对各类因素的综合 “效用值” 表示消费 者通过决策选择某一品牌将得到的消费满足大小。正常情况下, 消费者将选择带来最大效用的决策方案或 者效用决定的概率方案。互联网金融业的消费者效用函数与其效用影响因素之间存在函数关系, 当某一品 牌带给消费者的效用较其他品牌更多或者概率更大，消费者将选择该品牌。

我们假定互联网金融业存在 $\mathrm{n}$ 个品牌，各品牌都有各自特性的同时，也具有此类产品的共性。对其中 某一品牌而言, 从计量经济的角度能够观测到的品牌属性如金融风险、价格与收益、品牌传播、金融服务 
质量等因素, 采用 $\mathrm{x}_{\mathrm{i}}$ 来表示, 则 $\mathrm{n}$ 个品牌的可观测品牌属性记为向量 $\mathrm{x}_{\mathrm{i}}=\left(\mathrm{x}_{1}, \mathrm{x}_{2}, \cdots \mathrm{x}_{\mathrm{n}}\right)$; 用 $\mathrm{C}_{\mathrm{j}}$ 表示此品 牌的品牌传播状况; 用 $\mathrm{z}_{\mathrm{j}}$ 表示某品牌消费者 $\mathrm{j}$ 的消费选择特征, 其与多种因素有关, 例如消费者的上网熟 练程度、年龄、收入、受教育程度、消费习惯等, 即单个消费者所特有的消费偏好, 也是消费者选择的影 响因素, 是随机影响因子。本文认为, 互联网金融产品的产品属性、品牌传播及消费者个人偏好共同决定 互联网金融业消费者品牌选择行为。用效用函数表示为:

$U i j=u i j+\varepsilon i j=u(x i, z j, C j, \Theta)+\varepsilon i j$

上式中, $u_{i j}$ 是可以观测到的影响因素产生的效用, $\varepsilon_{i j}$ 则是不能观测到的因素产生的效用。由于效用 中的变量观测中存在误差、消费者偏好也在影响效用的大小加上有些变量本身就不适合观测的属性, 使得 品牌选择的消费者效用具体数值通常难以精确测度, 也缺乏事先预测的有效估计手段。为保证效用函数的 科学性, 要加入随机因子来修正效用函数, 将效用值作为随机变量。

综上可知, 互联网金融业消费者品牌选择的效用函数包含互联网金融产品属性和品牌传播两个基本要 素, 并假定研究的对象----互联网金融业产品属于彼此间具有较强替代性、产品特征也较相关的行业中的 消费者品牌选择, 调研数据代表的品牌特征能实现大部分都能被统计或观测到, 即实现产品属性、品牌传 播、消费者偏好的信息能被收集到，来判断、预测消费者的品牌选择行为。

考虑到互联网金融产品与传统金融产品的差异性, 消费者难以直接到达金融机构营业网点进行面对面 咨询和办理业务及售后, 消费者的品牌选择行为在很大程度上受产品属性和品牌传播效果的影响, 除了产 品本身属性外, 本文认为最重要的影响因素是互联网金融品牌的传播或者说是消费者感知到的品牌传播效 果。品牌传播成为影响互联网金融品牌消费者效用大小的一个重要因素。因此, 本文将品牌传播作为效用 函数的一个变量。因此, 消费者效用函数表达式可以表示成:

$\mathrm{U}=\mathrm{F}(\mathrm{x} 1, \mathrm{x} 2, \cdots, \mathrm{xm}), \mathrm{C} \in \mathrm{xi}$,

$\mathrm{x}_{\mathrm{i}}$ 表示互联网金融业消费者品牌选择时需考虑的效用影响因素, 其中品牌传播因素表示为 C。各因素 指标彼此相互独立, 各指标因素对消费者品牌选择影响的程度有差异时, 对各因素设置不同的权重系数: $\mathrm{U}=\sum_{i=1}^{m} w_{i} f_{i}\left(x_{i}\right)$, 式中 $\mathrm{m}$ 表示全部消费者效用影响因素, 当 $\mathrm{i}=1, \cdots, \mathrm{m}$ 时, 要考虑 $\mathrm{m}$ 个指标因素。各属性 的权重系数为 $\mathrm{w}_{\mathrm{i}}, \mathrm{f}\left(\mathrm{x}_{\mathrm{i}}\right)$ 为各个效用影响的函数, 把品牌传播因素从全部消费者效用影响因素中单列出来, 基于品牌传播的互联网金融业消费者选择行为效用函数可以表示成:

$$
U(Q, C)=\sum_{i=1}^{M} w_{i} Q_{i}+C
$$

式中表示消费者品牌选择的效用, $\mathrm{Q}$ 表示除品牌传播以外的效用影响因素; $\mathrm{w}_{\mathrm{i}}$ 是各效用因素的权重系 数, 每个效用因素对消费者的品牌选择带来的满足程度不同, 并将其取值设定为 $\mathrm{w}_{\mathrm{i}} \in[-1,1]$, 正负号表 示对消费者效用是呈正向还是负向作用; 同时认为品牌传播的因素对消费者效用影响最大, 并假设其传播 给消费者关于品牌的正面信息, 因此将其系数 $\mathrm{w}_{\mathrm{c}}$ 确定为 +1 , 故得出以上效用函数表达式。

\section{3 结语}

该文结合应用经济学与媒介经济学的相关理论，在对中国互联网金融业消费者选择行为分析的基础 
上, 从品牌传播、个人因素、宏观环境、微观环境、产品属性、广告劝说、群体参照、品牌服务等八个方 面归纳出影响消费者品牌选择行为的主要影响因素。以此为基础, 提出了消费者选择行为的效用函数, 指 出互联网金融产品的产品属性和品牌传播属性是最为重要的两个影响因素，从而构建了互联网金融业消费 者选择行为效用函数模型，为引导消费者品牌选择行为提供制定决策的参考和研究基础，为促进我国互联 网金融业健康快速发展提供消费者效用分析的研究支撑。

\section{致谢}

本文系湖南省教育厅科学研究项目课题（13C129）、湖南省社会科学成果评审委员会一般课题 （XSPYBZZ020）、湖南省哲学社会科学基金成果立项项目（16CGA005）阶段性成果。

\section{Acknowledgement}

This article is the stage result of the Hunan Provincial Education Department Science Research Project (13C129), Hunan Provincial Social Science Achievement Review Committee General Subject (XSPYBZZ020), Hunan Provincial Philosophy and Social Science Fund Project (16CGA005) .

\section{参考文献:}

[1] Bansal, H. S. andVoyer R. A. Word of mouth Processes wi thin a services Purchase decision context[J]. Journal of service research, 2000, 3(2):166-177.

[2] Gilly, M. C. , Graham, J. L. , Wolfinbarger, M. F. , andYale, L. J. A Dyadic Study of Interpersonal Information Search[J]. Journal of the Academy of Marketing Science, 1998, 2:83-100.

[3] Punj, G. N. andStaelin, R. A Model of Consumer Information Search Behavior for New Automobiles [J]. Journal of Consumer Researeh, 1983, 9(4):366-380.

[4] 王峥. 我国互联网金融的风险分析及防范措施 $[J]$. 时代金融, 2014, 08:82-83.

[5] 毛玫菁 大公信用数据有限公司分析师。互联网金融为什么需要信用信息教育普及 $[\mathrm{N}]$. 中国经济时 报, 2015-08-07012.

［6］本报记者 杨颖 霍玉菡. 首家互联网金融信用信息平台上线 [N]．中国贸易报, 2014-09-04006.

[7] 何健, 王永贵, 石贵成. 关系强度的前置因素及其绩效影响 [J]。管理世界, 2009, 05:180-181.

[8] 石贵成, 王永贵, 邢金刚, 于斌. 对服务销售中关系强度的研究——概念界定、量表开发与效度检验 [J]．南开管理评 论, 2005, 03:74-82.

[9] 郑宏明. 信任倾向与主观参照对网上购物意向的影响 [J]. 社会心理科学, 2007, Z2:121-127.

[10］王文松．消费者品牌信任及其营销对策[J]．河南科技大学学报(社会科学版), 2007, 03:86-88.

\section{References:}

[1] Bansal, H.S. and Voyer R.A. Word of mouth Processes within a services Purchase decision context [J]. Journal of service research, 2000, 3(2):166-177.

[2] Gilly, M.C.,Graham, J.L., Wolfinbarger, M.F. andYale, L.J.A Dyadic Study of Interpersonal Information Search[J].Journal of the Academy of Marketing Science,1998,2:83-100.

[3] Punj, G.N.andStaelin, R. A Model of Consumer Information Search Behavior for New Automobiles [J]. Journal of Consumer Researeh, 1983, 9(4):366-380.

[4] Wang Zheng. Risk Analysis and Preventive Measures of Internet Banking in China [J]. Time Finance, 2014, 08: 82-83. 
[5] Mao Meiqing Dagong credit Co. Data Analyst. Why Internet Banking Needs Credit Information Education [N]. China Economic Times, 2015-08-07012.

[6] Reporter Yangyin, Huo Yuhan. First Internet Financial Credit Information Platform on-line [N]. China Trade News, 2014-09-04006.

[7] He Jian, Wang Yonggui, Shi Guicheng. The Antecedents of Relationship Strength and Its Impact on Performance [J]. World Management, 2009, 05: 180-181.

[8] Shi Guicheng, Wang Yonggui, Xing Jingang, Yu Bin. Research on the Relationship Strength of Service Sales_Concept definition, scale development and validation [J]. Nankai Business Review, 2005, 03: 74-82.

[9] Zheng Hongming. The Influence of Trust Orientation and Subjective Reference on Online Shopping Intention [J]. Science of Social Psychology, 2007, Z2:121-127.

[10] Wang Wensong. Consumer Brand Trust and Its Marketing Strategy [J]. Journal of Henan University of Science and Technology (Social Science Edition), 2007, 03: 86-88.

Introduction to the author: Deng En(1978--), Male, Hunan University, Ph.D., associate professor of economics at Hunan Radio and Television University, research direction: Internet finance, financial economics; Correspondence Author: Zhao Ming(1978--)male, Hunan Radio and Television University economics lecturer, research direction: financial economy. 